\title{
A PRODUÇÃO SOBRE EDUCAÇÃO A DISTÂNCIA NO OPEN LEARNING: THE JOURNAL OF OPEN AND DISTANCE LEARNING - PERIÓDICO DA UNIVERSIDADE ABERTA DO REINO UNIDO
}

\author{
Ana Esmeralda Carelli \\ Maria Júlia Giannasi \\ Vilma Aparecida Gimenes da Cruz
}

\begin{abstract}
Resumo
Este estudo tem como objetivo analisar a produção de artigos do periódico Open Learning: the Journal of Open and Distance Learning, publicados no período de 1999 a 2003, para estabelecer uma síntese dessa produção com vistas a melhor compreender a realidade atual em âmbito internacional e oportunizar o compartilhamento das experiências na área como subsídio à pesquisa. O estudo em questão buscou identificar algumas variáveis como: autoria, abrangência geográfica, temática, tratamento do tema e a literatura utilizada na produção do artigo. Após este estudo constatouse que a autoria individual ainda é muito presente; aspectos relativos à tecnologia têm sido uma temática bem focalizada, porém com preocupação de maximizar o potencial da Educação a Distância; a abrangência geográfica dos artigos contempla mais os países onde a Open University é presente; os autores utilizam formas diversas para tratar seus temas, porém a mais marcante é o relato de experiência dos programas de EAD existentes nas diversas universidades. As referências de livros e de periódicos constituem-se nas fontes mais utilizadas pelos autores no periódico Open Learning. Destaca-se ainda que a Educação a Distância tem criado novas oportunidades educacionais, especialmente no nível superior.
\end{abstract}

\section{Palavras-chave}

Estudo de Produção - Open Learning; Estudo de Produção - Educação a Distância; Comunicação Científica - Educação a Distância 


\section{INTRODUÇÃO}

A sociedade do conhecimento pressupõe cidadãos com conjunto de competências e habilidades que ultrapassam a formação acadêmica convencional; o esperado é a formação ao longo da vida. Assim, a Educação a Distância (EAD) constitui-se em oportunidade para esta formação, e as tecnologias de informação e comunicação, possibilitam a ampliação das condições de oferta de cursos a distância, sobretudo pelo potencial da Web.

No cenário brasileiro, a Educação a Distância, ainda hoje, encontra resistência nos meios educacionais, mesmo considerando-se o destaque e a visibilidade já existentes. Com isto a produção científica nacional gerada nesta temática fica aquém do desejável, revestindo-se de fundamental importância buscar subsídios teóricos e pesquisas na área em outras realidades. Compartilhar experiências com instituições de ensino com tradição nessa modalidade de educação, como é o caso da Open University, criada na década de 70 e com grande expansão a partir do avanço das tecnologias de informação e comunicação (WANG; LIU, 2003), é fundamental. As diferenças regionais são significativas e, portanto, foram analisadas e consideradas neste estudo.

Como parte de pesquisa concluída no âmbito da realidade brasileira sobre Ensino a Distância no Brasil (CARELLI et al.,
2004) sentiu-se a necessidade de obter maiores subsídios em publicações estrangeiras, o que trouxe como resultado este estudo, de interesse para a comunidade envolvida com a temática da área; considerando-se que o periódico é o meio primário de disseminar os resultados de pesquisa e contribuir para o desenvolvimento científico. Considera-se que, "ao mesmo tempo que o pesquisador está envolvido no seu próprio trabalho e na aquisição de informações, está também produzindo e disseminando novas informações para os demais" (TARGINO, 2000, p. 80), salientando-se ainda o papel do periódico científico na divulgação de notas prévias e trabalhos em andamento.

Este estudo tem por objetivo, portanto, analisar a produção de artigos do periódico Open Learning (1985), publicados no período de 1999 a 2003, para estabelecer uma síntese dessa produção com vistas a melhor compreender a realidade atual e enfatizar a temática na literatura.

O periódico Open Learning: the Journal of Open and Distance Learning, é uma publicação da Open University, editado pela Carfax Publishing, parte do Taylor \& Francis Group. Existe no formato impresso e também disponível eletronicamente ${ }^{1}$ mediante assinatura. É um periódico de abrangência internacional no campo da aprendizagem aberta, flexível e a distância.

\footnotetext{
${ }^{1}$ http://www.tandf.co.uk/journals/carfax/02680513.html
} 
Aceita para publicação três tipos de artigos: que versem sobre assuntos teóricos e baseados em evidência, que reflitam o desenvolvimento desse tipo de aprendizagem, na área de educação e treinamento; que versem sobre assuntos práticos e que descrevam a implementação de sistemas de aprendizagem aberta, flexível e a distância; e, ainda, que sejam resenhas de livros (OPEN LEARNING, 1985).

\section{MATERIAL E MÉTODOS}

A escolha do periódico Open Learning deu-se em razão da quase inexistência de publicações especializadas em Educação a Distância no Brasil, além do mérito científico dessa revista, sobretudo levandose em conta a importância de compartilhar experiências na área de EAD, com outros países. Outro fator decisivo para a escolha foi a disponibilidade da coleção na Web, por um período de um mês, sem custos para os usuários interessados no acesso ao mesmo.

O periódico em questão, da Open University, é publicado quadrimestralmente. No período compreendido neste estudo, qual seja de 1999 a 2003, em que se disponibilizaram na Web, os volumes 14 a 18, foram analisados 87 artigos das seções de artigos teóricos e relatos de experiência, excluindo-se, dessa forma, as resenhas de livros. Ressalta-se ainda que não foram analisados os artigos compreendidos como editoriais.

As variáveis selecionadas para análise foram: autoria (individual ou coletiva), abrangência geográfica do artigo, ênfase temática, tratamento do tema e a literatura utilizada na produção do artigo.

\section{RESULTADOS}

Além dos aspectos selecionados para análise neste estudo, e que serão detalhados a seguir, destaca-se a relevância do periódico analisado para a área em questão e a importância de se conhecer a literatura estrangeira oriunda de países com considerável tempo de experiência em Educação a Distância.

Tem-se a autoria como uma variável importante de estudo na qualificação da produção científica, com destaque para a autoria coletiva, conforme destaca Oliveira (1999, p.57), ao afirmar que "os estudos de autoria múltipla caracterizam-se por maior possibilidade de extensão e aprofundamento do que aqueles de autoria única".

No entanto, quanto a esse aspecto, observou-se, curiosamente, que 52 artigos $(59,77 \%)$ são de autoria individual, representando quase $60 \%$ dos artigos analisados (conforme Tabela 1), contrapondo o que se observa na literatura científica em geral, em que a autoria coletiva reflete a comunidade científica, geralmente conce- 
bida em grupos (MEADOWS, 2000; SOLA virtuais de aprendizagem favorecem a auPRICE, 1976), e contrariando a tendência toria coletiva.

atual do E-learning, cujas comunidades

Tabela 1- Número de Autores por Artigo Publicado

\begin{tabular}{c|c|c}
\hline $\begin{array}{c}\text { Número de Autores } \\
\text { por Artigo }\end{array}$ & Número de Artigos & $\%$ \\
\hline 1 & 52 & 59,77 \\
2 & 26 & 29,88 \\
3 & 7 & 8,04 \\
4 & 1 & 1,14 \\
8 & 1 & 1,14 \\
\hline Total & $\mathbf{8 7}$ & $\mathbf{1 0 0}$ \\
\hline
\end{tabular}

No trabalho de Targino e Castro (2001, p. 47) abordando características dos periódicos eletrônicos, na área da saúde, também se constatou que a autoria individual ocupou a primeira posição, corroborando assim os dados deste estudo.

Na seqüência da análise, registrou-se 26 artigos escritos por dois autores $(29,88 \%)$; sete artigos escritos por três autores $(8,04 \%)$; um artigo com quatro autores $(1,14 \%)$ e também um artigo com oito autores $(1,14 \%)$.

Quanto à área geográfica, em que relacionam as experiências por países, ob- servou-se que em 24 artigos (27\%) não se referem ao país; em 23 deles mencionouse o Reino Unido $(26,43 \%)$ o que é compreensível, já que o periódico é daquele país, sabendo-se também que a Open University deve ser a maior produtora da literatura em razão da grande experiência e tradição na área. Dando continuidade à análise observa-se que as referências às áreas geográficas se diluem, havendo uma menção maior dos países onde está presente a Open University, conforme se pode observar na Figura 1. 


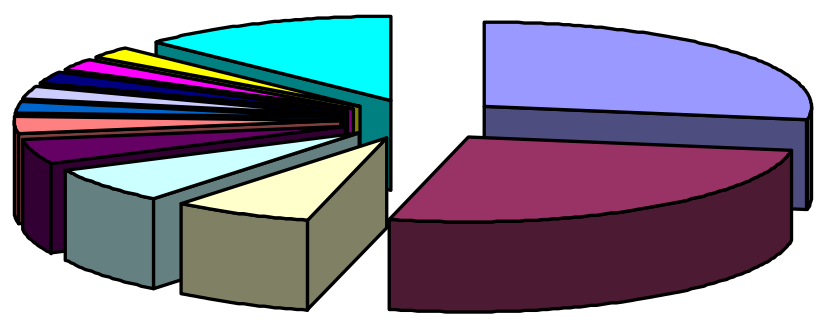

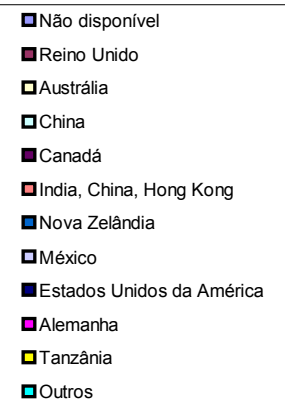

Figura 1 - Distribuição dos Artigos por Área Geográfica

O trabalho de Zhang e colaboradores (2005) evidencia este aspecto, qual seja, a relevância da presença da Open University na produção intelectual sobre EAD nos países de sua abrangência. Na investigação realizada na Open University of Hong Kong (OUHK), os autores examinaram a percepção dos alunos sobre o suporte tutorial on-line oferecido pelos 199 cursos dessa universidade. Cabe mencionar também o estudo realizado por Barbour (2005) que discute o avanço da Educação a Distância em Newfoundland e Labrador, províncias localizadas na costa Oeste do Canadá.

Quanto à ênfase temática dos artigos publicados, conforme se verifica no Quadro 1 , os recursos tecnológicos aparecem em primeiro lugar com 32 ocorrências. Podese constatar na literatura que essa é a tônica da produção na área em razão dos recentes avanços nas tecnologias de informação e comunicação que dão suporte para o desenvolvimento das políticas e práticas pedagógicas em EAD (JELFS; COLBOURN, 2002; KIRKUP; KIRKWOOD, 2005).

No artigo de Baggaley e Hoon (2005), é dada considerável importância à variável tecnologia; os autores focalizam os desafios enfrentados no uso da tecnologia na Educação a Distância pelos países asiáticos, em desenvolvimento.

Na seqüência da análise para esse estudo, destacam-se os mecanismos de aprendizagem, tais como suportes, estratégias de ensino, motivação etc., que visam o maior envolvimento do aluno com as tecnologias bem como com as propostas pedagógicas, com vistas ao aprendizado crítico e competente condizente com os 
desafios da sociedade atual. Registram-se nessa temática 19 artigos. Outros assuntos de menor ênfase podem ser visualizados no Quadro 1, por exemplo, Gestão em $E A D$, assunto de bastante interesse e importância na atualidade brasileira, que aparece em sete artigos. Em seguida, registram-se estudos tais como projetos pedagógicos, percepção do aluno, capacitação dos tutores e interatividade e, ainda os estudos sobre o perfil do aluno de EAD que parecem refletir também na produção brasileira.

Quanto ao tipo de trabalho que, conforme nosso entendimento é importante destacar, uma vez que retrata o olhar do autor sobre o tema em questão, observouse que relato de experiência foi predominante, aparecendo com 38 (43,66\%) do total dos artigos analisados no período, resultado que já era esperado em vista da tradição e acúmulo de experiência e conhecimento gerado nesses países. Pesquisa de campo aparece em seguida, com 33 artigos (37,93\%). Estudos teóricos aparecem em 16 artigos (18,39\%), sendo os estudos históricos englobados nessa categoria.

\begin{tabular}{|l|c|}
\hline \multicolumn{1}{|c|}{ Ênfase temática dos artigos } & Número de Artigos \\
\hline Recursos tecnológicos & 32 \\
Mecanismo de aprendizagem do aluno & 19 \\
Gestão de Educação à Distância & 7 \\
Projeto pedagógico & 6 \\
Percepção do aluno & 5 \\
Capacitação de tutor ou de pessoa ou de professor & 5 \\
Interatividade & 5 \\
Perfil do aluno / Habilidades e competências de alu- & 5 \\
nos na Educação à Distância & \\
Potencial da Educação à Distância e democratiza- & 4 \\
ção do ensino & 3 \\
Estudos comparativos & 3 \\
Educação à Distância como Educação Continuada & 2 \\
Interface: Tecnologia/Projeto pedagógico/Indivíduo & 2 \\
Estudos teóricos & 2 \\
Avaliação da aprendizagem ou da proposta pedagó- & 1 \\
gica & \\
Produção de material para a Educação à Distância & 1 \\
Avaliação de projetos e propostas de Educação à & 1 \\
Distância & \\
Metodologia de pesquisa & \\
\hline
\end{tabular}

Quadro 1 - Ênfase Temática dos Artigos Publicados

O respaldo científico que os autores para produção dos seus artigos é visualirecebem por meio da literatura utilizada zado nas referências bibliográficas citadas 
e, dessa forma, torna-se um aspecto relevante da análise. Em relação ao respaldo científico, os resultados (Tabela 2) apontam para o artigo de periódico citado em primeiro lugar, em 77 artigos, com 781 ocorrências; seguido pelo livro, citado em 76 artigos, com 531 ocorrências, e capítulos de livros que aparecem em 295 citações em 70 artigos, característica essa marcante na área de Educação, ou seja, muita citação de livros e capítulos, quando em outras áreas prevalece a citação de artigos de periódicos. Também é característico dos alunos de pós-graduação da área de Educação utilizar muito mais a literatura publicada em livros do que a de periódicos ou de outros suportes de informação, conforme trabalho de Okiy (2003).

Ao retomar a análise dos temas apontados no Quadro 1, observa-se que a tecnologia está prevalecendo, o que reforça a utilização dos artigos de periódicos como principal fonte de citação na área e, sobretudo, porque o periódico é a principal fonte de informação para o desenvolvimento da educação e da ciência.

Tabela 2 - Tipos de Documentos Citados nos Artigos Analisados

\begin{tabular}{l|c|c|c}
\hline $\begin{array}{c}\text { Tipos de documentos } \\
\text { citados }\end{array}$ & $\begin{array}{c}\text { Número de } \\
\text { Artigos }\end{array}$ & $\begin{array}{c}\text { Número de } \\
\text { referências }\end{array}$ & $\%$ \\
\hline Artigo de periódico & 77 & 781 & $38,74 \%$ \\
Livro & 76 & 531 & $26,34 \%$ \\
Capítulo de livro & 70 & 295 & $14,63 \%$ \\
Trabalho de evento & 52 & 130 & $6,45 \%$ \\
Documento eletrônico & 25 & 62 & $3,08 \%$ \\
Relatório & 27 & 43 & $2,13 \%$ \\
Não identificado / Referên- & & & $1,39 \%$ \\
cias incompletas & 21 & 28 & $1,29 \%$ \\
Material instrucional & 17 & 26 & $1,24 \%$ \\
Tese & 14 & 25 & $1,19 \%$ \\
Material interno & 4 & 24 & $1,14 \%$ \\
Artigo de jornal & 11 & 23 & $1,09 \%$ \\
Documento oficial & 13 & 22 & $0,50 \%$ \\
Comunicação pessoal & 2 & 10 & $0,40 \%$ \\
Outros & 7 & 8 & $0,20 \%$ \\
Trabalho não publicado & 2 & 4 & $0,10 \%$ \\
Resenha de livros & 1 & 2 & $0,10 \%$ \\
Resumo de tese & 1 & 2 & $100,00 \%$ \\
\hline TOTAL & & $\mathbf{2 . 0 1 6}$ & \\
\hline
\end{tabular}

\section{CONSIDERAÇÕES FINAIS}

Como suporte para a revisão de literatura no âmbito da EAD foi significativa a presente análise, porque permitiu trazer elementos da literatura estrangeira como reforço para a área de EAD.
Considera-se ainda que este estudo, não obstante seja uma síntese parcial, permitiu não só agregar novas informações e possibilitar o conhecimento de outras realidades em diferentes contextos socioeconômicos, educacionais e culturais, como 
também vislumbrar temáticas para novas pesquisas, expandindo a visão do potencial da EAD e de sua representação na sociedade do conhecimento.

No contexto da Educação a Distância em que a tecnologia é, hoje muito usada, facilitando e contribuindo de forma inegável para a inclusão educacional, não se pode deixar de considerar como significativo suporte para os projetos pedagógicos, o olhar crítico sobre essa tecnologia. A tecnologia deve adequar-se ao projeto pedagógico de tal forma que se possa explorar todo o seu potencial, a imensa gama de oportunidades que proporciona a todos os sujeitos do processo (conteudistas, professores, tutores, monitores, alunos, entre outros) em benefício da qualidade do ensino e aprendizagem e não o contrário, ou seja, o projeto pedagógico adequar-se à tecnologia.

$\mathrm{Na}$ aprendizagem do adulto (andragogia) no complexo meio das comunidades virtuais de aprendizagem, a ênfase deve estar na qualidade e interatividade efetiva, e a tecnologia, conquanto seja o meio para viabilizar propostas concretas e inovadoras de EAD, não pode ser o recurso principal da aprendizagem do adulto.

No entanto, relatos de projetos pedagógicos ainda são incipientes na literatura e não descrevem de maneira abrangente como se devam usar adequadamente os recursos tecnológicos em benefício do aprendizado. Os relatos de experiências, se bem que em maioria significativa no periódico analisado, não detalham os projetos pedagógicos em sua essência, antes, dão mais ênfase ao uso da tecnologia.

\section{REFERÊNCIAS}

BAGGALEY, Jon; HOON, Maria Ng Lee. PANdora's box: Distance learning. Learning, Media and Technology. v.30, n.1, p.5-14, mar. 2005.

BARBOUR, Michael. From telematics to web-based: The progression of distance education in Newfoundland and Labrador. British Journal of Educational Technology, v. 36, n.5, p. 1055-58, 2005.

CARELLI, Ana Esmeralda; GIANNASI, Maria Júlia; CRUZ, Vilma Aparecida Gimenes da; VALENTE, Silza Maria Pasello; HIDALGO, Lúcia Amaral. Ensino a Distância no Brasil: discurso ou prática? Diagnóstico da situação. Londrina: UEL, 2004.

JELFS, A.; COLBOURN, C. Virtual seminars and their impact on the role of the teaching staff. Computers and Education, v.38, p.127-36, 2002.

KIRKUP, Gill; KIRKWOOD, Adrian. Information and communications technologies (ICT) in higher education teaching: a tale of gradualism rather than revolution. Learning, Media and Technology, v.30, n.2, p.185-99, Jul. 2005.

MEADOWS, A.J. Avaliando o desenvolvimento da comunicação eletrônica. In: MUELLER, S.P.M.; PASSOS, E.L.J. (ORG. . . Comunicação científica. Brasília: UnB, 2000. p.23-34.

OKIY, Rose B. A citation analysis of education dissertations at the Delta State University, Abraka, Nigeria. Collection Building, v. 22, n. 4, p. 158-161, 2003. 
OLIVEIRA, Maria Helena Mourão de. Leitura e escrita: análise da produção com ênfase no universitário. Campinas, 1999. Tese. (Doutorado em Psicologia) - PUCCampinas, 1999.

OPEN LEARNING. London: Carfax Publishing, 1985. v. 14-18

SOLLA PRICE, D.J. O desenvolvimento da ciência. Rio de Janeiro: Livros Técnicos e Científicos, 1976.

TARGINO, Maria das Graças. Comunicação científica: uma revisão de seus elementos básicos. Informação \& Sociedade: Estudos, João Pessoa, v. 10, n. 2, p. 37$85,2000$.

. CASTRO, Mônica Maria Machado Ribeiro Nunes. Perfil dos títulos e artigos dos periódicos do grupo de publicações eletrônicas em medicina e biologia (Grupo e-pub). Revista de Biblioteconomia de Brasília, v. 25, n.1, p. 27-57, jan/jun 2001.

WANG, C.; LIU, Z. Distance education: basic resources guide. Collection Building, v.22, n.3, p. 120-130, 2003.

ZHANG, Wei-yuan; FERRIS, Kirk; YEUNG, Lesley. Online tutorial support in open and distance learning: students' perceptions. British Journal of Educational Technology, v.36, n.5, p. 789-804, 2005.

\section{Ana Esmeralda Carelli}

Docente da Universidade Estadual de Londrina carelliana@uel.br

Maria Júlia Giannasi

Docente da Universidade Estadual de Londrina mjulia@uel.br

Vilma Aparecida Gimenes da Cruz Bibliotecária da Universidade Norte do Paraná gimenes@dilk.com.br

\section{Title}

The production of Distance Education in the Open Learning: The Journal Of Open And Distance Learning - Journal of Open University of United Kindon

\section{Abstract}

This study aims at analyzing the production of Open Learning: the Journal of Open and Distance Learning articles published from 1999 to 2003, to have an overview of the current trend of distance education at international level so to share experience and to provide bases for other researches. This study identified several variables such as: authorship, geographical range, topics, methodology and the literature used in the article production. This study identified that the individual authorship is still very used; technology has been a very well focused theme with concern maximizing its educational potential in distance learning; geographical ranges noticed is directly related to countries where Open University is found; the authors use several methodologies to develop their researches, the most outstanding topic is experience report of the distance education programs in the several universities. The main bibliographic references are books and journals. An important finding is that distance education has provided new education opportunities, especially at university level.

\section{Keywords}

Production Studies - Open Learning; Production Studies - Distance Education; Scientific Communication - Distance Education

\section{Título}

La producción sobre educación a la distancia en el Open Learning: The Journal of Open and Distance Learning - Periódico de la Universidad Abierta del Reino Unido

\section{Résumen}

Este estudio tiene como objetivo analizar la producción de artículos del periódico Open Learning: the Journal of Open and Distance 
Learning, publicados en el período de 1999 a 2003, para establecer una síntesis de esa producción con vistas para mejor comprensión de la realidad actual al nivel internacional y hacer oportuno el compartimiento de las experiencias en el área como un soporte a la investigación. El estudio en pauta buscó identificar algunas variables como: autoría, ámbito geográfico, temática, tratamiento del tema y la literatura utilizada en la producción del artículo. Después de este estudio se puede constatar que la autoría individual todavía está muy presente; aspectos relacionados a la tecnología había sido una temática bien focalizada, aunque con la preocupación de maximizar el potencial de la educación a la distancia (EAD); el ámbito geográfico de los artículos contempla más los países donde la Open University está presente; los autores utilizan formas distintas para tratar sus temas, pero los temas más destacadas son los relatos de experiencias de los programas de EAD existentes en las distintas universidades. Las referencias de libros y de periódicos se constituyen en las fuentes más utilizadas por los autores en el periódico Open Learning. Además se destaca que la educación a la distancia había creado nuevas oportunidades educacionales, especialmente al nivel superior.

\section{Palabras Clave}

Estudio de Producción - Open Learning; Estudio de Producción - Educación a la Distancia; Comunicación Científica - Educación a la Distancia 